\title{
Electrode Movement Prevention - an Animal Model
}

\author{
V. MASOPUST ${ }^{1}$, I. PETRÍKOVÁ ${ }^{2}$, M. MLČEK ${ }^{3}$, J. HOLUBOVÁ $^{4}$, R. ROKYTA $^{5}$, \\ J. FRICOVÁ ${ }^{5}$, K. JANOUŠKOVÁ ${ }^{4}$
}

${ }^{1}$ Department of Neurosurgery and Neurooncology, First Faculty of Medicine, Charles University, Prague, Czech Republic, ${ }^{2}$ Department of Applied Mechanics, Faculty of Mechanical Engineering, Technical University of Liberec, Liberec, Czech Republic, ${ }^{3}$ Department of Physiology, First Faculty of Medicine, Charles University, Prague, Czech Republic, ${ }^{4}$ Department of Otorhinolaryngology, Central Military Hospital, Prague, Czech Republic, ${ }^{5}$ Department of Normal, Pathological and Clinical Physiology, Third Faculty of Medicine, Charles University, Prague, Czech Republic

Received August 6, 2018

Accepted February 18, 2019

Epub Ahead of Print June 6, 2019

\section{Summary}

Electrode migration is the most common complication of spinal cord stimulation (SCS). The problem of longitudinal migration has already been solved, but lateral migration remains the most common current complication. The present article describes new electrodes fixation opportunities for the reduction of lateral migration in SCS. The pig was chosen as an animal model to illustrate a new protocol of electrode fixation for the control of lateral and longitudinal migration. The displacement of the electrode was measured using two different optical methods: the digital image stereo-correlation and the digital image processing methods. Fixation with two anchors has always considerably reduced electrode displacement and when fixation is done with two anchors and a loop then lateral migration is reduced by $62.5 \%$ and longitudinal migration is reduced by $94.1 \%$. It was shown that the results are significantly different at the $a=0.001$ significance level. Based on a statistical evaluation it is possible to state that the differences between experimental results obtained for three different protocols of lead fixation are statistically significant and we can recommend the new fixation method for common practice.

\section{Key words}

Spinal cord stimulation • Electrode movement prevention • Pig model

\section{Corresponding author}

Masopust Václav, Department of neurosurgery and neurooncoogy, First Faculty of Medicine, Charles University, U vojenské nemonice 1200, Prague 6, 16200 Czech Republic. E-mail: masopust.vaclav@gmail.com

\section{Introduction}

Neuromodulation is the process in which several classes of neurotransmitters in the nervous system regulate diverse populations of neurons, but neuromodulation in neurosurgery is the process in which artificial devices alter the function of the nervous system. One of the neuromodulation methods for the treatment of chronic pain is spinal cord stimulation (SCS). Electrode migration is the most common complication of SCS and is more frequent with percutaneous electrodes than with paddle-type ones. Electrode migration may be compensated by stimulator reprogramming, but if this fails, surgical repositioning is mandatory (Bendersky and Yampolsky 2014). The most common complication that requires a revision surgery in $23 \%$ of patients is electrode displacement (Andersen, 1997). Wire migration is hard-ware complications. SCS is associated with several complications. Hardware-related complications are common in $38 \%$ of cases and this includes wire migration in $22.6 \%$ (Makhail et al. 2011). In the present article a new electrodes fixation is described to reduce wire migration complications. Two anchor fixation options and three different types of lead fixation are discussed in this work.

\section{Material and Methods}

One pig (Landrace, 53kg) was used for all 
experiments. Prior to the study, the animal was housed in an accredited conventional University animal facility with natural daylight, free access to water, feed twice daily by a mixture recommended for young swine. Ambient conditions (temperature, air) were regulated according to relevant recommendations. Animals were kept in groups of up to four. The pig was anesthetized using a standard general anesthesia procedure. After $24 \mathrm{~h}$ of fasting, anesthesia was induced by midazolam $(0.3$ $\mathrm{mg} / \mathrm{kg}$ IM) followed by ketamine hydrochloride (15 to 20 $\mathrm{mg} / \mathrm{kg}$ IM). Anesthesia was continued with initial propofol and morphine boluses, $(2 \mathrm{mg} / \mathrm{kg} \mathrm{IV}$ and 0.1 to $0.2 \mathrm{mg} / \mathrm{kg} \mathrm{IV}$, respectively) and animals were orotracheally intubated. Continuous IV infusion of propofol (8 to $10 \mathrm{mg} / \mathrm{kg} / \mathrm{h}$ ) combined with morphine ( 0.1 to $0.2 \mathrm{mg} / \mathrm{kg} / \mathrm{h}) \mathrm{IV}$ were used to maintain anesthesia, the depth of which was regularly assessed by photoreaction and corneal reflex. Anesthesia was provided during the whole study. Mechanical ventilation was maintained throughout the study in volume control regime set to $\mathrm{Vt} 8 \mathrm{ml} / \mathrm{kg}$, PEEP $5 \mathrm{~cm} \mathrm{H}_{2} \mathrm{O}, \mathrm{FiO}_{2} 0.25$, $\mathrm{I}: \mathrm{E} 1: 2$, rate adjusted to keep $\mathrm{pCO}_{2} \quad 5.0-5,5 \mathrm{kPa}$ (34-41 $\mathrm{mm} \mathrm{Hg}$ ). Stimulating electrodes were inserted through the fascia and the muscle (musculus longissimus dorsi) up to the cutaneous surface to simulate the passage of an electrode in the real environment. The electrode passed through the fascia and the muscle and the slave followed at a distance of $10 \mathrm{~cm}$. The lead was fixed in the incision before entry into the spine by one of the methods listed above (Figs 1 A, B, C). Fixation was carried out to the fascia in all cases. The second end of the lead was tunneled from the side of the pig and fastened to a rope. The rope passing over a pulley was loaded with a weight of $1 \mathrm{~kg}$, causing the movement of the wire. The rope was loaded gradually over the course of 15 seconds. Porcine respiratory movements were eliminated (artificial ventilation) during the measurement. The displacement of the electrode was measured and evaluated by two different optical methods (Sun et al. 2002):

- Digital image stereo-correlation method

- Digital image processing method

\section{Digital image stereo-correlation method}

Measurement and evaluation were carried out by a ISTRA4Dantec Dynamics optical correlation device which allows non-contact measurement of the threedimensional displacements of points on the surface of a body. One white target with random pattern of black dots was attached to the electrode and the second one on
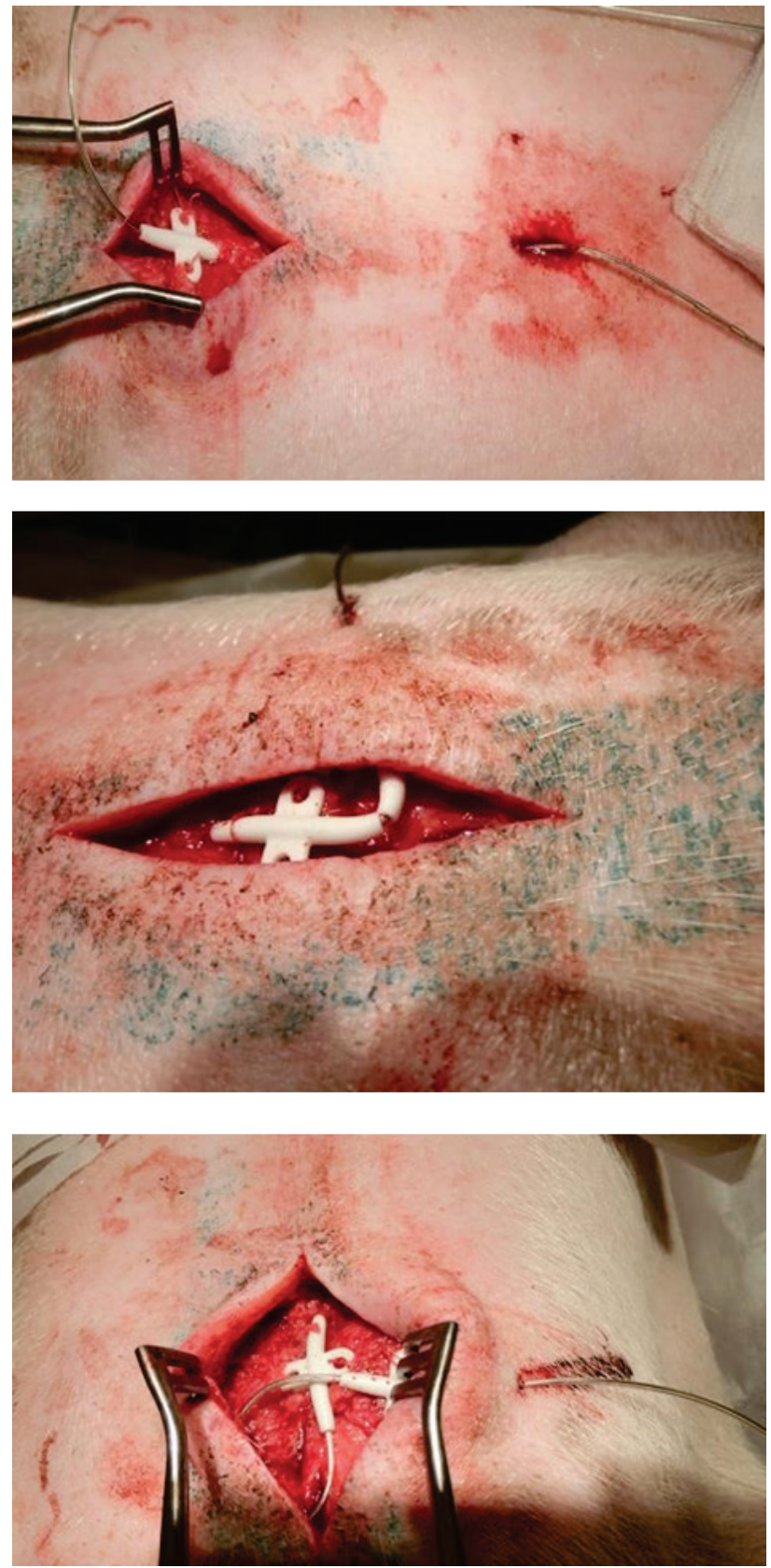

Fig. 1. (A) Electrode fixed by one anchor, (B) Electrode fixed by two anchors, (C) Electrode fixed by two anchors with a loop

the skin of the pig (Fig. 2). The targets moved slowly during the loading of the wire. The images of the moving targets were recorded simultaneously by two cameras with a sampling frequency of 17 frames per second (Fig. 3). The recorded images were then processed with the ISTRA4 software and the 3D coordinates $(x, y$ and $z$ ) of the target points were determined. The three components of displacement $\Delta x, \Delta y$ and $\Delta z$ were calculated as the difference of coordinates between the actual and the reference images. The example of total displacement evaluation is illustrated in Fig. 4. The 
electrode with its target lies freely on the dorsal part of the pig and therefore the movement of the target is a general spatial motion. The total displacement $d$ of the end of the electrodes is described by the equation

$$
d=\sqrt{ }(\Delta x)^{2}+(\Delta y)^{2}+(\Delta z)^{2} .
$$

Measurement of electrode displacement by means of digital image processing (DIP)

The measurement was focused on precise determination of the displacement of the electrode in the longitudinal direction of the spine. A small glass tube with an internal diameter of $3 \mathrm{~mm}$ was fixed with sutures onto the dorsal skin of the pig parallel to the spine. The pseudo-stimulation wire was passed through the epidural space, up to the body surface of the pig and inserted into the tube (Fig. 5). Two targets were glued onto the surface of the tube with a distance of $100 \mathrm{~mm}$ between them ensuring a fixed benchmark permanent photographic image. The electrode could move freely in the tube axis direction while its movement in the transverse direction was limited by the tube wall. The wire was loaded in the same way as previously described. In each experimental session, two digital images of the electrode position were recorded with a resolution of $4608 \times 3456$ pixels. The first image was recorded before any load application (starting position) and the second one was recorded in steady state at the end of loading.

The pixel coordinates of both targets and the labelled end of the electrode were determined in each image with the Matlab Image Processing Toolbox. The coordinates correspond to places with the maximum correlation coefficient between matrix $A$ containing the grayscale intensities of rectangular area around the targets or around the electrode end and matrix B of the same size picked from every image using detailed scanning. The correlation coefficient $r$ is defined by

$$
r=\frac{\sum_{m} \sum_{n}\left(A_{m n}-\bar{A}\right)\left(B_{m n}-\bar{B}\right)}{\sqrt{\left(\sum_{m} \sum_{n}\left(A_{m n}-\bar{A}\right)^{2}\right)\left(\sum_{m} \sum_{n}\left(B_{m n}-\bar{B}\right)^{2}\right)}},
$$

where $\bar{A}$ and $\bar{B}$ are the means of values in matrices $A$ and $B$. The distance of the targets in pixel units in every image was used for conversion between pixels and millimetres. The targets distance was $100 \mathrm{~mm}$, or about 2811 pixels, respectively, and therefore the measurement accuracy of the position of the electrode is higher than $0.1 \mathrm{~mm}$.

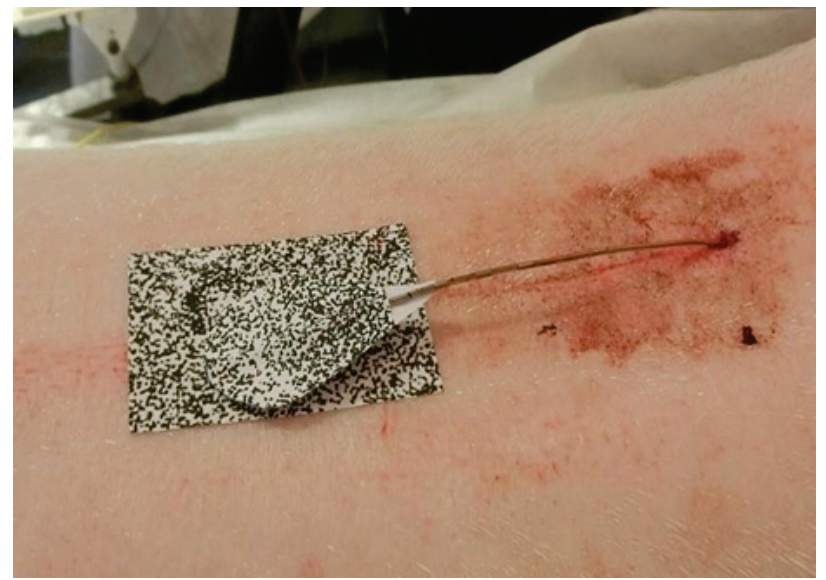

Fig. 2. Target with random pattern of black dots

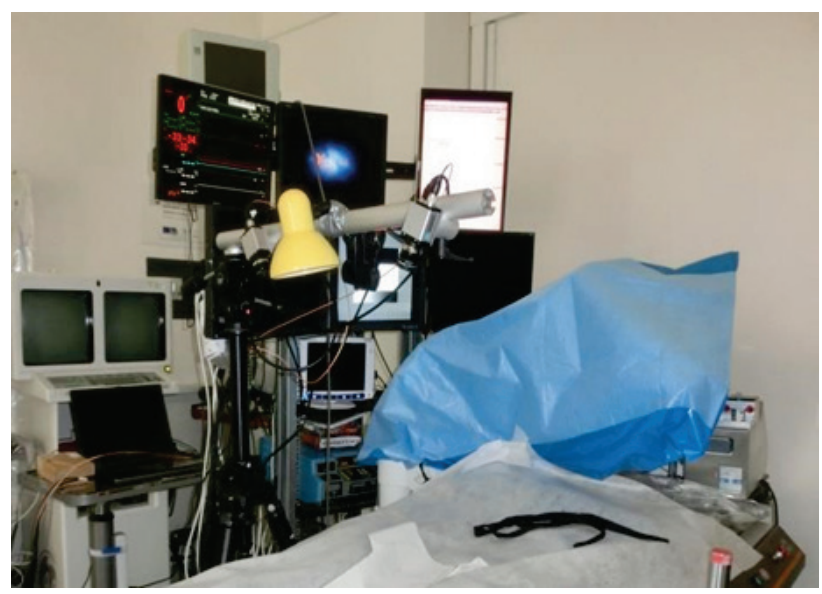

Fig. 3. Digital image stereo-correlation system with two cameras

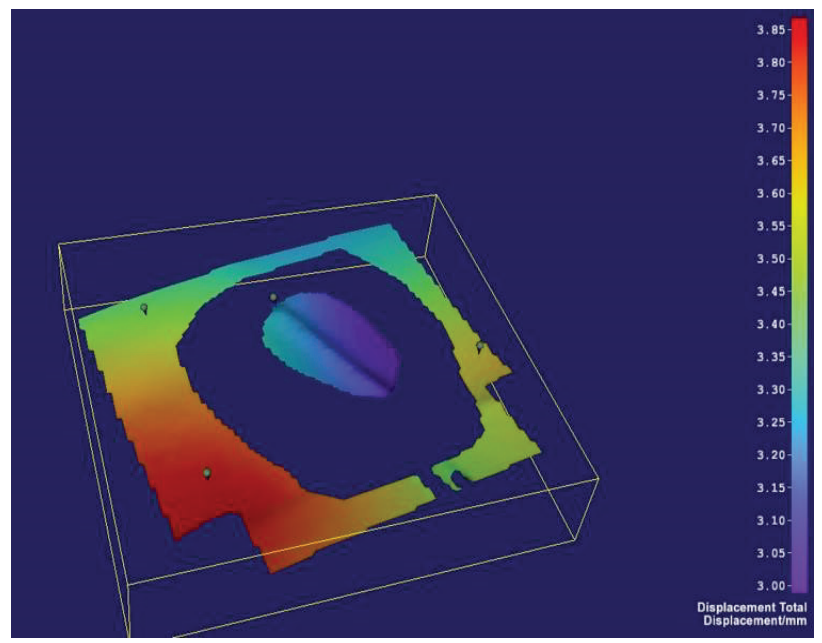

Fig. 4. Example of total displacement evaluation

\section{Results}

\section{Digital image stereo-correlation method}

Three different ways of wire anchoring were chosen for comparison of the electrode movement. The 
influence of the different ways of anchoring the wire is illustrated on (Fig. 6). where the total displacement $d$ during the loading are displayed. The electrode displacement depends markedly on the anchoring lead, namely the number of anchors and the type of lead anchoring. It is evident that fixation of the lead by two anchors with a loop between them significantly reduces the total displacement of the electrode, i.e. the lateral migration of the electrode was reduced by $62.5 \%$.

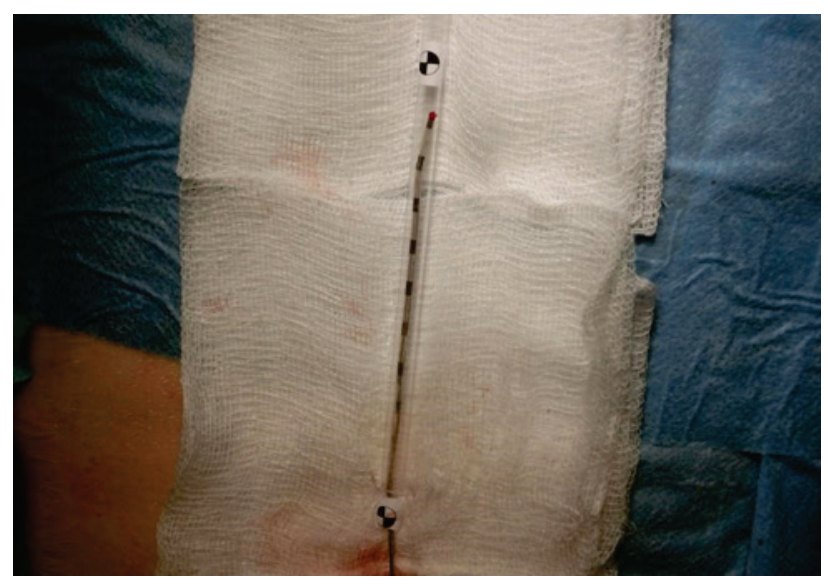

Fig. 5. Experimental setting of the electrode displacement (DIP)

Measurement of electrode displacement by means of digital image processing (DIP)

The position of the electrode end was determined in each image and its displacement of the electrode was calculated as the difference between its positions before and after loading. The results of the three sets of measurements are presented in (Fig. 7). and in Table 1. It is evident that the method of wire anchoring is critical in avoiding displacement of the electrode due to

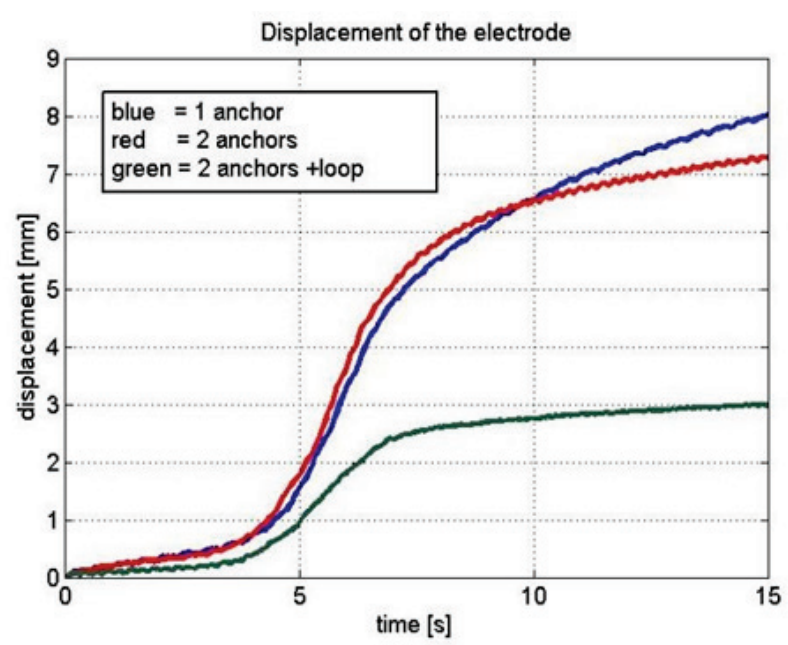

Fig. 6. Total displacement of the end of electrode. sudden patient movement (longitudinal migration).

Exploratory data analysis (EDA) was conducted within statistical analysis of the results and the parametricity was verified by the Shapiro-Wilk test. Subsequently analysis of variance (ANOVA) was used to assess the significance of the difference between electrode displacements measured for the three types of fixation. It was shown that the results are significantly different at the $\alpha=0.001$ significance level.

\section{Discussion}

Military University Hospital is a large neuromodulation centre in Czech Republic. The major part of neuromodulation therapy in Czech Republic is performed there. We have many years of experience. The first neuro-stimulation of SCS in Czech Republic was performed at our department in 1973 (Beneš 1973). A new type of electrode fixation was used with two anchors and a loop in our department over the last two years. We have had no complications with electrode migration since we started using the new fixation method. This is why we wanted to demonstrate the importance of the new fixation method in laboratory conditions.

The most common type of neuromodulation is the stimulation of SCS. In the world literature the terms neuromodulation and neuro-stimulation are often confused or published for the same concept (Rokyta and Fricova 2012).

The main indication for neuro-stimulation is failed back surgery syndrome (FBSS). In more than $85 \%$ of cases, neurostimulation is performed in patients with FBSS (Masopust, 2014). One of the causes of FBSS is epidural fibrosis (EF) (Braverman et al. 2011, Coskun

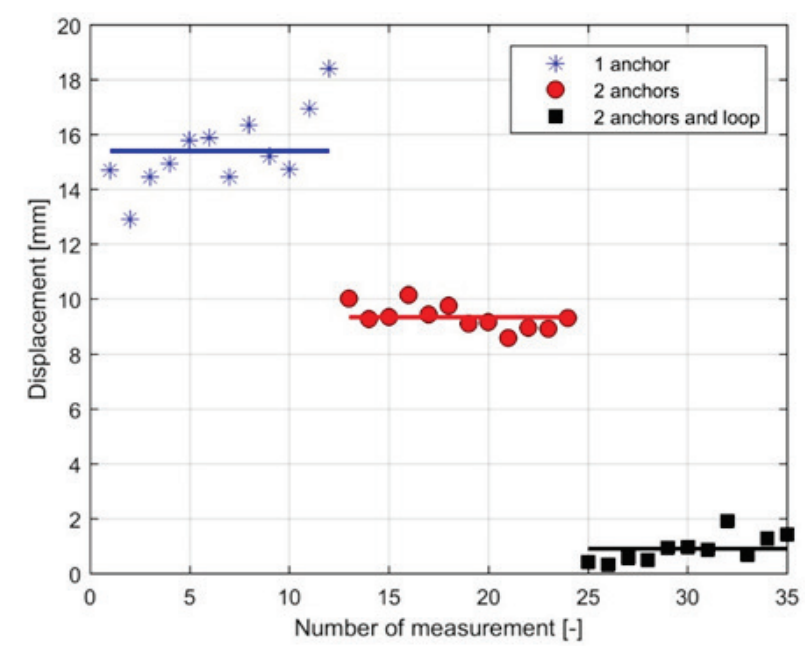

Fig. 7. Displacement of electrode for different anchoring wires. 
Table 1. Displacement of electrode for different anchoring wires

\begin{tabular}{lccc}
\hline Way of anchoring wire & 1 anchor & 2 anchors & 2 anchors and loop \\
\hline Number of measurements & 12 & 12 & 11 \\
Average displacement [mm] & 15.41 & 9.35 & 0.92 \\
Standard deviation $[\mathrm{mm}]$ & 1.41 & 0.46 & 0.48 \\
\hline
\end{tabular}

et al. 2000). Although neuromodulation remains the most widely used method for treatment of refractory pain at FBSS, there is no evidence of the greater effectiveness of SCS versus alternative treatments (Turner et al. 2010). Neuromodulation may still be beneficial for carefully classified patients (Waszak et al. 2017).

Our patients who are indicated for neuromodulation have MRI-proven EF in more than $90 \%$ of cases (Masopust et al. 2014). EF behaves as a reparative inflammation with characteristic symptoms and clinical course (pain) (Masopust et al. 2007). FBSS is a major cause of chronic neuropathic pain. FBSS affects more than $40 \%$ of patients who undergo spinal surgery for low back pain (Lad et al. 2014). The relationship between EF and FBSS has been widely discussed in the algesiological literature. Especially, the role of EF in the development of FBSS is discussed, as is its prevention and treatment. Clinically significant EF is described in the literature as between $5 \%$ and $33 \%$ (Frish et al. 1996, Cinottis et al. 1998). If the patient is predisposed to EF, we assume that the moving activity of the electrodes is low for scarring of electrodes. But it is true that EF does not affect electrode fixation due to a long period of scarring and electrode migration was the most common complication for our patients.

SCS is associated with many complications. Hardware-related complications are common in $38 \%$ of cases (Makhail et al. 2011). These complications included electrode displacement in $22.6 \%$ of cases (Makhail et al. 2011). Electrode migration is the most common complication of SCS. An electrode shift occurs more often for percutaneous electrodes than for paddle type ones (Bendersky and Yampolsky 2014).

We can sometime solve the electrode movement by neuro-stimulation reprogramming but this solution is not always possible and surgical repositioning is necessary in $23 \%$ of cases (Andersen 1997).

A special case is deep brain stimulation, where there is the possibility of fixation to the bone (Contarino et al. 2013).

Other complications are very rare. These complications include epidural hematoma and a dura injury with cerebrospinal fluid leakage (Eldrige et al. 2006, Franzini et al. 2005). The rarest complication is epidural abscess (Rauchwerger et al. 2008). We can influence these complications and minimize them with the correct insertion angle and a smooth introduction. "Midline anchoring" is used (Mironer et al. 2004).

But why is the migration of wires so frequently a complication? Many types of anchors were manufactured when neuromodulation therapy became the standard medical method. Now we can use a firmer anchoring system in practice. The result is a stronger connection between electrode and anchor. But today we know that fixation between the anchor and electrode is not the cause of electrode movement. Unfortunately, there exists the problem of the biological response of tissues. The tissues are different for each individual. Collagen is the most abundant structural protein to be found in the extracellular matrix. These proteins have mechanical and supportive functions in a wide range of connective tissues (Sun et al. 2002).

The elasticity of a tissue is determined by the type of collagen monomer and its occurrence. And it is precisely the elasticity of the tissue that is the first cause of the instability and electrode shift. The second cause of instability is a fixation at one point between an anchor and a fascia. The fixation is done on both sides of the anchor. The anchor with three silicone wings was made and recommended as a prevention of anchor movement (Kumar et al. 2007). More point fixations cannot help us with our problem of the movement of an electrode because fixations are done between an electrode and a fascia near each other. The displacement can be reduced by fixing the anchor to the deep fascia firmly and supplemented by using of silicone glue and by placing the pulse generator on an abdominal wall instead of the gluteal region (Kumar et al. 2007). However, although these recommendations are beneficial, they cannot change the number of complications resulting from physical laws. When the bar is fixed in one place, then no number of fixations can prevent the bar from moving in 
a circle. If we want to impede the movement of the rod, we have to make two fixations in two places sufficiently far apart. We can only use these methods if the bar is rigid. When the rod is flexible then the fixation points cannot be placed into one plane, otherwise the rod bends between fixations and we are not able to impede movement of the distal end. This conclusion should be validated in an animal model. We need evidence for the hypothesis that the displacement of the electrode depends markedly on the anchoring wire, namely the number of anchors and the manner of the anchoring wire. And for that we did three models. The first model was with one anchor, the second model was with two anchors close to each other and the last and third was a model with two anchors with a loop between them (Petrikova et al. 2017).

Two types of electrode migration are possible. The first type is lateral migration and the further type is longitudinal migration. A change in electrode position on a dura surface without the possibility of reprogramming is created by lateral migration. Longitudinal migration is important in order to avoid a progressive ejection of electrodes. Longitudinal migration was resolved as early as 2006 (Renard and North, 2006). Lateral migration remains the most common complication today. An animal model was used to demonstrate the importance of our new model of fixation for lateral migration.

On the animal model, the electrode was implanted through the same tissue as in humans, this means through the skin, subcutaneous fat, fascia and muscle. The rope trajectory was the same as in our patients, it means at right angles to the electrodes because in this way the rotational movement of the body is simulated. The respiratory movements of our porcine were eliminated during the measurement. That was needed if we wished to attain maximum reality. An animal model was anaesthetized using standard general anaesthesia means.

It was evident from our measurements that electrode fixation by two anchors with a loop between them significantly reduces the total displacement of the electrode i.e. lateral migration. Our measurement was made by the digital image stereo-correlation method.
Lateral migration of the electrode was reduced by $62.5 \%$.

Using digital image processing, it is evident that the method of anchoring wire is critical in order to avoid the displacement of the electrode due to sudden patient movement. Lateral migration of the electrode was reduced by $94.1 \%$ It was shown that results are significantly different at the $\alpha=0.001$ significance level.

Results which were obtained by both measuring methods demonstrate the difference between the tested types of anchoring wires. Fastening with two anchors significantly reduced the displacement of the electrodes. The electrode almost does not move when fixation is performed with two anchors and a loop. The average shift was less than $1 \mathrm{~mm}$.

Based on a statistical evaluation it is possible to state that the differences between the experimental results obtained for three different types of cable fixation are statistically significant.

\section{Conclusions}

Results obtained by both measuring methods demonstrate the difference between the tested types of anchoring wires.

Fixation with two anchors has always considerably reduced electrode displacement and when fixation is performed with two anchors and a loop then lateral migration is reduced by $62.5 \%$ and longitudinal migration is reduced by $94.1 \%$.

Based on statistical evaluation it is possible to state that the differences between experimental results obtained for three different types of cable fixation are statistically significant and we can recommend the new fixation method for common practice.

\section{Conflict of Interest}

There is no conflict of interest.

\section{Acknowledgements}

This paper was supported by Charles Univesity grant Q35 and the grant MO 10-12.

\section{References}

ANDERSEN C: Complications in spinal cord stimulation for treatment of angina pectoris. Differences in unipolar and multipolar percutaneous inserted electrodes. Acta Cardiol 52: 325-333, 1997.

BENDERSKY D, YAMPOLSKY C: Is spinal cord stimulation safe? A review of its complications. World Neurosurg 82: 1359-1368, 2014. 
BENEŠ V, ŠLÉGR Z, STRNAD M: Electrostimulation of the dorsal spinal cord for the treatment of pain. (In Czech) Cas Lek Cesk 113: 1565-1568, 1973.

BRAVERMAN DL, SLIPMAN CW, LENROW DA: Using gabapentin to treat failed back surgery syndrome caused by epidural fibrosis: A report of 2 cases. Arch Phys Med Rehabil 82: 691-693, 2001.

CINOTTIS G, ROYSAM GS, EISENSTEIN SM, POSTACCHINI F: Ipsilateral recurrent lumbar disc herniation. A prospective, controlled study. J Bone Joint Surg Br 80: 825-832, 1998.

CONTARINO MF, BOT M, SPEELMAN JD, DE BIE RM, TIJSSEN MA, DENYS D, BOUR LJ, SCHUURMAN PR, VAN DEN MUNCKHOF P: Postoperative displacement of deep brain stimulation electrodes related to wireanchoring technique. Neurosurg 73: 681-688, 2013.

COSKUN E, SÜZER T, TOPUZ O, ZENCI, M, PAKDEMIRLI E, TAHTA K: Relationships between epidural fibrosis, pain, disability, and psychological factors after lumbar disc surgery. Eur Spine J 9: 218-223, 2009.

ELDRIGE JS, WEINGARTEN TN, RHO RH: Management of cerebral spinal fluid leak complicating spinal cord stimulator cord stimulator implantation. Pain Pract 6: 285-288, 2006.

FRANZINI A, FERROLI P, MARRAS C, BROGGI G: Huge epidural hematoma after surgery for spinal cord stimulation. Acta Neurochir (Wien) 147: 565-567, 2005.

FRISH EW, HEISEL J, RUPP S: The failed back surgery syndrome: reasons, intraoperative findings, and long-term results: a report of 182 operative treatments. Spine 21: 626-633, 1996.

KUMAR K, WILSON JR: Factors affecting spinal cord stimulation outcome in chronic benign pain with suggestion to improve success rate. Acta Neurochir Suppl 97: 91-99, 2007.

LAD SP, BABU R, BAGLEY JH, CHOI J, BAGLEY CA, HUH BK, UQILIWENEZA B, PATIL CG, BOAKYE M: Utilization of spinal cord stimulation in patients with failed back surgery syndrome. Spine (Phila Pa 1976) 39: 719-727, 1976.

MAKHAIL NA, MATHEWS M, NAGEEB F, GUIRGUIS M, MEKHAIL MN, CHENG J: Retrospective review of 707 cases of spinal cord stimulation: indications and complications. Pain Pract 11: 148-153, 2011.

MASOPUST V, HÄCKEL M, FRICOVÁ J: Use of hylase in the treatment of postoperative epidural fibrosis. (In Czech) Bolest 4: 207-210, 2007.

MASOPUST V, ROKYTA R, BENEŠ V: Neuromodulation. (In Czech) Cesk Slov Neurol N 110: 138-152, 2014.

MIRONER EY, BROWN CH, SATTERTHWAITE RJ, COHEN M, TONDER LM, Grumman S: A new technique of "midline anchoring" in spinal cord stimulation dramatically reduces lead migration. Neuromodul 7: 32-37, 2004.

PETRIKOVA I, MARVALOVA B, CIRKL D, MASOPUST V: Measurement of displacement of percutaneous lead for spinal cord stimulation. EAN 2016 - 54th International Conference on Experimental Stress Analysis, 2017.

RAUCHWERGER JJ, ZOARSKI GH, WAGHMARAE R, RABINOWITZ RP, KENT JL, ALDRICH EF, CLOSSON CW: Epidural abscess due to spinal cord stimulator trial. Pain Pract 8: 324-328, 2008.

RENARD VM, NORTH RB: Prevention of percutaneous electrode migration in spinal cord stimulation by a modification of the standard implantation technique. J Neurosurg Spine 4: 300-303, 2006.

ROKYTA R, FRICOVÁ J: Neurostimulation methods in the treatment of chronic pain. Physiol Res 61 (Suppl 2): S23-S31, 2012.

SUN Y, LUO Z, FERTALA A, AN K: Direct quantification of the flexibility of type I collagen monomer. Biochem Biphys Res Commun 295: 382-386, 2002.

TURNER JA, HOLLINGWORTH W, COMSTOCK BA, DEYO RA: Spinal cord stimulation for failed back surgery syndrome: outcomes in a workers' compensation setting. Pain 148: 14-25, 2010.

WASZAK PM, MODRIĆ M, PATUREJ A, MALYSHEV SM, PRZYGOCKA A, GARNIER H, SZMUDA T: Response to: clinical use, quality of life and cost-effectiveness of spinal cord stimulation used to treat failed back surgery syndrome. Asian Spine J 11: 677-678, 2017. 